\title{
In silico evaluation of potential inhibitory activity of remdesivir, favipiravir, ribavirin and galidesivir active forms on SARS-CoV-2 RNA polymerase
}

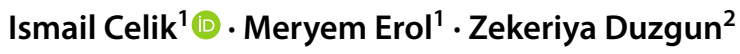

Received: 30 November 2020 / Accepted: 21 March 2021 / Published online: 25 March 2021

(c) The Author(s), under exclusive licence to Springer Nature Switzerland AG 2021

\begin{abstract}
Since the outbreak emerged in November 2019, no effective drug has yet been found against SARS-CoV-2. Repositioning studies of existing drug molecules or candidates are gaining in overcoming COVID-19. Antiviral drugs such as remdesivir, favipiravir, ribavirin, and galidesivir act by inhibiting the vital RNA polymerase of SARS-CoV-2. The importance of in silico studies in repurposing drug research is gradually increasing during the COVID-19 process. The present study found that especially ribavirin triphosphate and galidesivir triphosphate active metabolites had a higher affinity for SARS-CoV-2 RNA polymerase than ATP by molecular docking. With the Molecular Dynamics simulation, we have observed that these compounds increase the complex's stability and validate the molecular docking results. We also explained that the interaction of RNA polymerase inhibitors with $\mathrm{Mg}^{++}$, which is in the structure of NSP12, is essential and necessary to interact with the RNA strand. In vitro and clinical studies on these two molecules need to be increased.
\end{abstract}

\section{Graphic abstract}

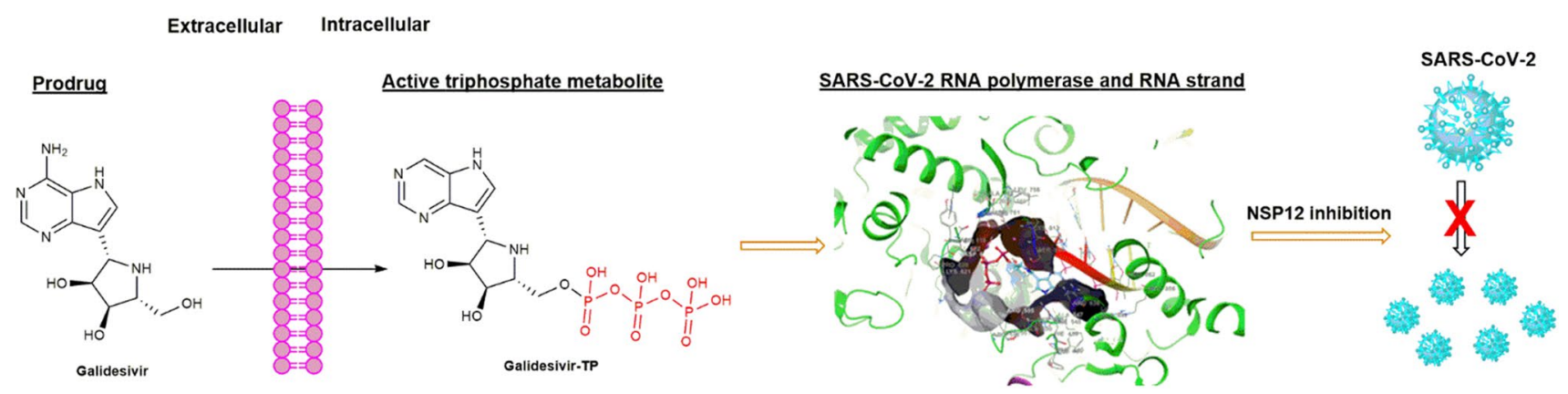

Keywords SARS-CoV-2 $\cdot$ Galidesivir $\cdot$ Ribavirin $\cdot$ Molecular docking $\cdot$ Molecular dynamics

Ismail Celik

ismailcelik@erciyes.edu.tr

1 Department of Pharmaceutical Chemistry, Faculty of Pharmacy, Erciyes University, Kayseri, Turkey

2 Department of Medical Biology, Faculty of Medicine, Giresun University, Giresun 28100, Turkey

\section{Introduction}

Coronavirus disease 2019 (COVID-19) is a viral infection with high pathogenicity and contagiousness caused by Severe Acute Respiratory Syndrome Coronavirus 2 (SARS-CoV-2). First, cases of pneumonia of unknown etiology were reported on November 17, 2019, in Wuhan, Hubei Province, China [1]. On January 7, 2020, it was identified that the disease agent was a new coronavirus (2019-nCoV) that had not been detected in humans before. The was later named coronavirus disease-19 (COVID-19). 
In one study examining the 2019 new CoV genome, this new virus was found to have $89 \%$ nucleotide homology with bat SARS-like CoVZXC21 and $82 \%$ with human SARS-CoV BJ01 2003. Hence, it was named SARS-CoV-2 because of its close resemblance to SARS-CoV [2]. The co-crystal structure of 2019-nCoV protease, 6LU7, has $\sim 99 \%$ identity with SARS-CoV protease [3]. SARS-CoV-2 is a single-stranded, positive polarity enveloped RNA virus. COVID-19 spread rapidly to many countries and was officially declared a pandemic by the World Health Organization on March 11, 2020, having caused the deaths of more than 4000 people $[4,5]$. The disease is highly contagious, and at the onset of the disease, the main symptoms were fatigue, fever, dry cough, myalgia, and dyspnea. Less common symptoms were nasal congestion, headache, runny nose, sore throat, vomiting, and diarrhea. The virus can be found in patients' respiratory secretions 1-2 days before the onset of clinical symptoms and two weeks after the disease symptoms. The incubation period of the disease is between 4 and 6 days. Severe cases usually have dyspnea and/or hypoxemia one week after onset and then go on to septic shock, followed by Acute Respiratory Distress Syndrome (ARDS) [6-8]. It has been shown that this disease is transmitted from person to person, especially adults, who are susceptible to COVID-19, and the severity of the disease is related to age. It has also been shown that the disease is more severe in people with comorbidities such as hypertension, diabetes, and cardiovascular disease [9]. The fact that infected people can infect others without showing any symptoms also makes it difficult to control COVID-19.

Coronaviruses are $80-220 \mathrm{~nm}$ in diameter and contain a single-stranded RNA of approximately $30 \mathrm{kbs}$ in length as nucleic acid [10]. A study using the genetic sequencing method reported a $96 \%$ similarity between the gene sequence of SARS-CoV-2 and bat coronavirus [11]. Coronaviruses attach to host cells via the spike (S) protein on the outer surface and enter the cell; $\mathrm{S}$ protein recognizes the receptor in the target cell and regulates the entry of the virus into the host cell. The virus's life cycle begins with binding the $S$ protein to the angiotensin-converting enzyme 2 (ACE2) receptor on the host cell surface. It has been shown that SARS-CoV-2 also enters the host cell using similar mechanisms after binding to the ACE2 receptor $[12,13]$. Structural studies revealed that SARS-CoV-2 spike (S) glycoprotein binds ACE2 with higher affinity $(\sim 15-40 \mathrm{nM})$ than SARS$\mathrm{CoV}$ spike protein $[14,15]$. Details of the pathological mechanisms leading to multisystemic organ dysfunction in SARS-CoV-2 infection are as yet unknown $[12,16]$. The treatments currently used in COVID-19 are the treatments applied to prevent the virus's entry into the cell-viz. inhibit or reduce its replication, and suppress the increased inflammation response in line with this physiopathogenesis
[17]. Also, convalescent plasma treatment, which includes infected and recovered patients' antibodies, is among the options [18].

Coronaviruses are one of the few RNA viruses that have a genomic regulation mechanism. The development of nucleoside analogs effective against coronaviruses has been particularly difficult due to a unique exoribonuclease (ExoN). ExoN functions as a corrective enzyme that corrects errors in the growing RNA chain [19]. The RdRp (RNA-dependent RNA polymerase) nonstructural protein 12 (NSP12) is highly conserved among coronaviruses, making it an attractive target for broad-spectrum antiviral drugs. The RdRp enzyme allows the viral genome to be copied into new RNA copies using the host cell's mechanism. Those in adenine or guanine analogs target the RdRp and block viral RNA synthesis across a broad spectrum of RNA viruses, including human coronaviruses. Generally, the rate-limiting step for activation of nucleoside analogs is the production of the nucleoside monophosphate. Nucleoside phosphoramidites such as remdesivir, favipiravir, ribavirin are bioisosteres of monophosphates and bypass this rate-limiting step. However, nucleoside phosphoramidites should be administered as prodrugs to mask the charged phosphonate group and allow faster cell entry. The nucleoside analog inhibits the viral RNA-dependent RNA polymerase (RdRp) by competing with the usual counterpart ATP, GTP, UTP, or CTP [20, 21]. Therefore, favipiravir, remdesivir, ribavirin, and galidesivir are thought to be potential drugs against SARS-CoV-2 [22-25].

Prodrugs are inactive drug forms designed by chemical changes in the original drug to increase the utilization of drugs in the human body. It is called a prodrug because after it is taken into the body by any means, it metabolizes kidney, liver, stomach acid, intestinal alkalinity (basicity) and changes its chemical structure. It creates a drug with a different structure, reaching the target tissue and having therapeutic properties. Even if prodrug drugs enter the blood for any reason, if they do not change, they do not show a pharmacological effect and have no therapeutic effect [26]. By way of example, Oseltamivir phosphate is a prodrug that is rapidly and extensively hydrolyzed in vivo to its active metabolite, oseltamivir carboxylate, which is a potent and selective inhibitor of influenza A and B virus neuraminidase [27]. Likewise, ribavirin, galidesivir, favipiravir, and remdesivir are in prodrug form and are converted into active metabolites after being taken into the body.

Ribavirin (tribavirin) is a broad-spectrum guanosine analog antiviral prodrug widely used to treat hepatitis C, RSV (respiratory syncytial virus) infection, CrimeanCongo hemorrhagic fever, and hantavirus. For the effect of SARS-CoV-2, high doses of 1.2 to $1.4 \mathrm{~g}$ orally every $8 \mathrm{~h}$ are required, which causes serious side effects in the patient in terms of hematological and hepatotoxic side 
effects potential. Inhalation administration also showed no advantage over oral or intravenous administration. It is also not recommended for pregnant women due to its teratogenic activity [28]. Clinical studies have shown that ribavirin can work synergistically with interferon $\beta$ and inhibit SARSCoV replication (Fig. 1). The poor in vitro activity of ribavirin against coronaviruses has been attributed to its removal by ExoN [29].

Galidesivir (BCX4430) is a broad-spectrum antiviral prodrug by BioCryst, a pharmaceutical company based in Durham that has potential in the treatment of COVID-19 and is safe and well-tolerated in Phase 1 trials in healthy subjects [30, 31]. Phase 1 clinical safety and pharmacokinetic studies of galidesivir by both intravenous and intramuscular administration have been completed in healthy volunteers. Currently, Galidesivir is under phase 2 human trial for Coronavirus in Brazil and around the world. Galidesivir has demonstrated broad-spectrum activity in vitro with EC50 ranging from $\sim 3$ to $\sim 68 \mu \mathrm{M}$ in preclinical studies against more than 20 RNA viruses in nine different families-filoviruses, togaviruses, bunyaviruses, arenaviruses, paramyxoviruses, flaviviruses, coronaviruses [31, 32]. Initially developed for hepatitis $\mathrm{C}$ virus, it phosphorylates Galidesivir, an adenosine nucleoside analog that inhibits viral RNA polymerase into a triphosphate that mimics the cellular kinases ATP. Viral RNA polymerases incorporate the drug's monophosphate nucleotide into the growing RNA chain, resulting in early chain termination, thereby disrupting the activity of viral RNA-dependent RNA polymerase [33, 34] (Fig. 1).

Favipiravir is a broad-spectrum prodrug that is phosphoribosylated inside the cell to form the active metabolite favipiravir-ribofuranosyl-5'-triphosphate. T-705-RTP competes with purine nucleosides and interferes with viral replication by incorporating into the viral RNA, potentially inhibiting RNA-dependent RNA polymerase ( $R d R p)$ of RNA viruses [20, 22, 35, 36]. It affects the RdRp of the influenza virus with a semi-maximal inhibitory concentration of $0.022 \mu \mathrm{g} / \mathrm{mL}$. Still, it does not affect the human DNA polymerase $\alpha, \beta$, and $\gamma$ subunits up to $100 \mu \mathrm{g} / \mathrm{mL}$. In some clinical studies conducted in the patient group receiving favipiravir, shorter viral clearance time, a higher recovery rate in chest imaging significantly reduced fever reduction and cough relief time in ordinary COVID-19 patients and patients with hypertension/or diabetes. Immediate approval of favipiravir (formulation: tablet, $0.2 \mathrm{~g}$ ) for a clinical trial in adult patients with novel coronavirus pneumonia was announced by the National Medicines Administration (NMDA) in China in March 2020 because it has been observed to exhibit good overall efficacy and safety with minimal side effects [37].

Remdesivir (RDV, formerly GS-5734) is a monophosphoramidate nucleoside analog prodrug with broad-spectrum action against various DNA and RNA viruses initially developed in response to the 2014-2016 Ebola epidemic in West Africa [3, 19, 24, 28]. RDV changes from its pro-form to the active triphosphate form that inhibits RdRp by non-essential RNA chain termination. The newly formed structure is an adenosine analog attached to viral RNA chains and leads to premature termination [23, 38-40]. The exoribonuclease of the virus, which usually reads and corrects replication errors, cannot work against the active form of remdesivir. Remdesivir is more active against viruses lacking ExoN but may partially escape correction and maintain strong antiviral activity in the presence of ExoN [19]. Recently, the FDA granted an

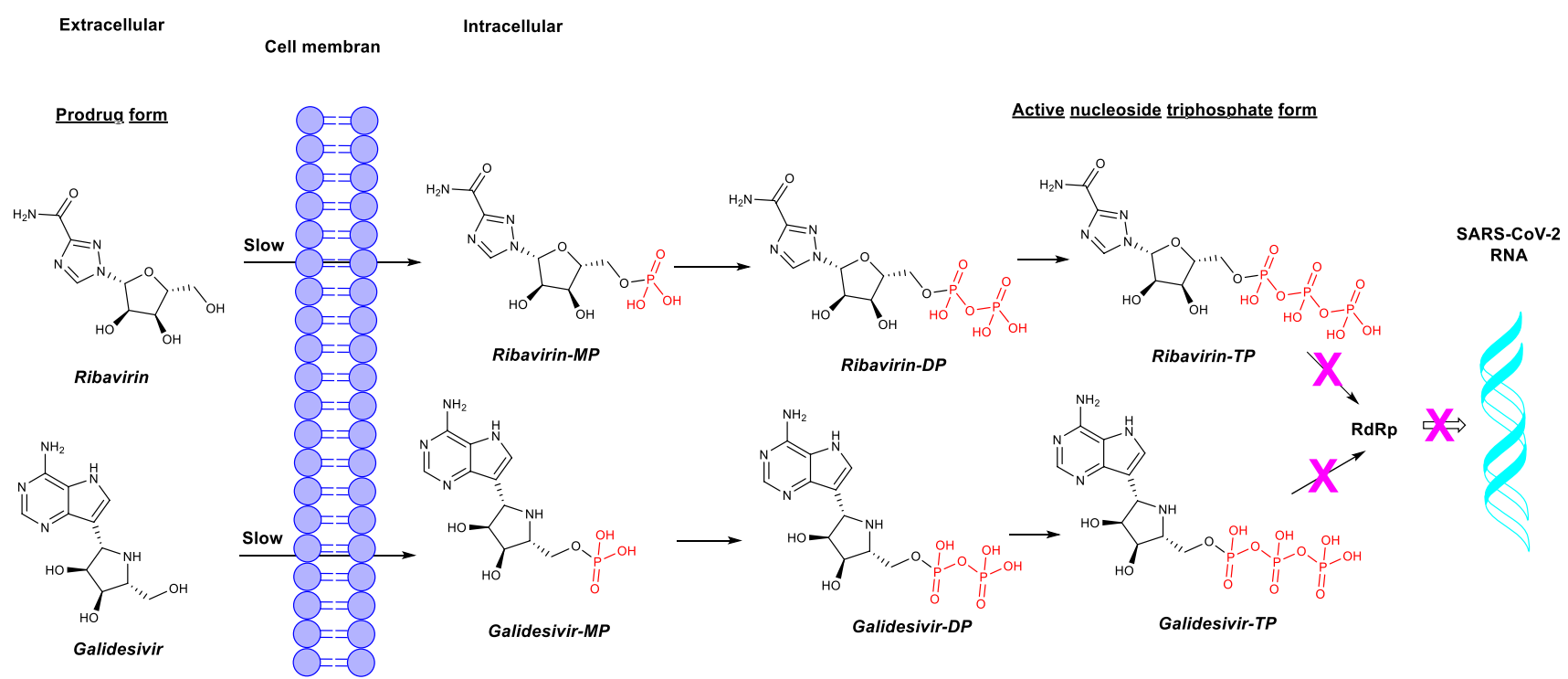

Fig. 1 The entry into the cell of the antiviral drugs ribavirin and galidesivir and the formation reaction of the active triphosphate forms 
emergency use permit for Remdesivir for potential COVID19 treatment [41]. Because the drug is highly selective for the virus's RNA polymerase, it is expected that humans' toxic side effects will be low. Thanks to its long half-life, it provides ease of use once a day. The therapeutic dose used for COVID-19; in the form of intravenous administration of $200 \mathrm{mg} /$ day on the first day and $100 \mathrm{mg}$ /day on the following days for 10 days. When the remdesivir treatment results are evaluated with clinical studies, 53 cases (patients with 94\% oxygen saturation or receiving oxygen support) were followed for at least 28 days using remdesivir at the dose mentioned above. While $68 \%$ of the cases showed improvement in terms of oxygen support, $15 \%$ got worse. Of 30 intubated patients, 57\% were extubated, and ECMO (extracorporeal membrane oxygenation) application could be terminated in three of four patients connected to ECMO (extracorporeal membrane oxygenation). After 28 days of follow up, the cumulative incidence of clinical improvement was evaluated as $84 \%$. Mortality was $13 \%$ after treatment was completed. Serious side effects were encountered in $23 \%$ of the cases. The most common side effects; ALT elevation was noted as diarrhea, rash, renal dysfunction, and hypotension [42].

In this study, we aimed to analyze the interaction pathway of the active forms of ribavirin, galidesivir, favipiravir, and remdesivir prodrug antiviral drugs in the SARSCov-2 RNA polymerase active site by molecular docking and dynamic studies. Unlike many other computational studies, we carried out our study by considering the interaction of active forms of drug molecule candidates with both NSP12 and RNA strand. Unfortunately, many in silico studies have focused on inactive prodrug forms of drug candidates [43-47], and these studies are unlikely to be successful. Likewise, in many computational studies, classical discourse includes the statement that other structures other than NSP12 (NSP7, NSP8, and RNA strand) were deleted [46, 48]. Still, RNA polymerase inhibitors must interact with both the NSP12 and the RNA strand for activity.

\section{Material method}

\section{Homology modeling}

In the NSP12-NSP7-NSP8 complex (PDB: 7BV2), missing residues $51-83,101-117$, and 896-910 were detected in the nsp12 chain and reconstructed according to homology modeling. The missing residues were added with the homology modeling tool included in the Chimera software regarding the protein sequence in the 7BV2 PDB file $[49,50]$.

\section{Molecular docking}

Molecular docking studies were carried out with the Schrödinger Maestro module [51-53]. Firstly, the SARSCoV-2 RNA-dependent RNA polymerase (RdRp) enzyme (PDB: 7BV2) crystal structure was imported from the protein data bank (http://www.rcsb.org/pdb/) into the 'Protein Preparation Wizard' module. Hydrogens were added, nonbonding command with metals, the formation of disulfide bonds, deleting water at $5 \AA$ distance from het groups, and preprocess by creating pH: $7.00 \pm 2.00$ het states using Epik. Subsequently, water molecules and $\mathrm{Zn}^{++}$and $\mathrm{Mg}^{++}$metals contained in protein crystal structures were retained, and remdesivir monophosphate and POP molecules other than the SARS-CoV-2 NSP7, NSP8, NSP12 RNA polymerase protein, and RNA structure were deleted. Finally, $\mathrm{H}$ bond determination was optimized using PROKA pH: 7.00 with water sample orientation and the complex structure was prepared by minimizing OPLS3 force fields.

Galidisevir, ribavirin, remdesivir, favipiravir drug molecules, and ATP were downloaded in 3D SDF file format from PubChem (https://pubchem.ncbi.nlm.nih.gov/). Those prodrugs' metabolite was drawn, minimized, and saved as an SDF file using Chem3D 17.01. Formed compounds were entered into the 'Ligprep' module. The OPLS3 force field was preferred, and ionization was carried out using Epik in the range of possible $\mathrm{pH}: 7.00 \pm 2.00$, desalinated, and tautomer-formed. Chirality from the 3D structure of ligands was determined and prepared. The complex structure's active site coordinates were prepared based on the remdesivir monophosphate in the 7BV2 structure in the size $x$ : $91.517, y: 92.382, z: 103.734$, and $25 \times 25 \times 25 \AA^{3}$ using the 'Receptor Grid Generation' module. Finally, the calculation of theoretical ligand-protein-RNA interactions was performed with Extra Precision (XP) using flexible ligand options of the 'Glide Ligand Docking' module. The Glide score and Glide emodel were evaluated, and 2D/3D interactions of ligand and protein were determined and exhibited.

\section{Molecular dynamics simulation}

The Glide docking tool's conformation was obtained in the Schrodinger suite to be located in the protein's active center. Compounds were extracted with Chimera software (Pettersen et al. 2004). Ligand topology parameters were obtained in accordance with the Amber force field using the AM1-BCC semi-empirical quantum calculation method with ACPYPE software [54, 55].

All simulations were run with GROMACS 2020.3 software [56]. The "Leap Frog" integration was used, and 2 fs time steps were applied. The system was prepared in periodic boundary conditions (PBC) as "Rhombic Dodecahedron." Cube sizes were calculated at a distance of $1.2 \mathrm{~nm}$ from 
the corner of the protein-ligand complex. Amber99SB-ildn force field and "TIP3P" water model were preferred [57]. The system was neutralized with $0.15 \mathrm{mM} \mathrm{Na}-\mathrm{Cl}$. Energy minimization was performed by applying the 50,000 step Steepest Descent algorithm. To bring the system to equilibrium, 300 ps NVT, and $1 \mathrm{~ns}$ NPT simulations were performed. According to the NVT stage, all bonds and atomic positions were restricted at $1000 \mathrm{~kJ} / \mathrm{mol}$ force in the LINCS algorithm. In NPT simulation, only bonds were fixed with LINCS Restriction Algorithm. All simulations were performed at $2 \mathrm{fs}$ time intervals. Verlet was used as the cut-off scheme during the production phase, and the Nose-Hoover algorithm was used as the temperature coupling algorithm. The temperature was set to $300 \mathrm{~K}$. The Perinello-Rahman algorithm with isothermal compressibility was preferred as the pressure coupling algorithm, and the pressure was set to one atmosphere. All restrictions were removed, and a $50 \mathrm{~ns}$ long MD simulation was performed for each protein compound complex. "Particle-mesh-Ewald" was used as a long-range electrostatic algorithm. Cut-off of Van Der Waals and short-term electrostatic interactions were set at $10 \AA$.

\section{Results}

\section{Molecular docking}

The crystal structure of SARS-CoV-2 RNA polymerase consists of NSP12 (A), NSP8 (B), NSP7 (C) chains, and RNA strand. There are two-piece $\mathrm{Zn}^{++}$ions and two-piece $\mathrm{Mg}^{++}$ metal ions in the NSP12 structure. ATP, which activates RNA polymerase, and interacts with $\mathrm{Mg}^{++}$(1004 numbered) by forming a salt bridge [58]. As the RNA-NSP12 complex and remdesivir interaction were examined, it was observed that the first phosphate group of remdesivir created an attractive charge with $\mathrm{Mg}^{++} 1004$ and 1005. While it was seen that the 20th uracil of RNA formed a covalent bond with the same phosphate group, it was observed that the same uracil base formed a pi-pi interaction with the bicyclic ring. It was observed that while Arg555 interacted with the bicyclic structure with an attractive charge, the 10th uracil of RNA formed hydrogen bonds with the nitrogen atoms of the hexane ring of the bicyclic structure. Also, it was observed that the remdesivir and the Val557, Cys622, Asp623, Ser682, Ala688, Thr687, Asn691, and Asp760 residues contacted with Van Der Waals interactions. According to molecular docking results, galidesivir, remdesivir, and ribavirin (except favipiravir) prodrugs form a salt bridge with $\mathrm{Mg}^{++} 1004$. When binding energies were examined, galidesivir $(-6.187 \mathrm{kcal} / \mathrm{mol})$, galidesivir triphosphate $(-8.994 \mathrm{kcal} / \mathrm{mol})$, ribavirin $(-6.128 \mathrm{kcal} / \mathrm{mol})$, ribavirin triphosphate $(-9.280 \mathrm{kcal} / \mathrm{mol})$, remdesivir $(-7.555 \mathrm{kcal} /$ $\mathrm{mol})$, remdesivir triphosphate $(-9.278 \mathrm{kcal} / \mathrm{mol})$, favipiravir
$(-5.336 \mathrm{kcal} / \mathrm{mol})$, favipiravir triphosphate $(-8.951 \mathrm{kcal} /$ $\mathrm{mol})$, ATP $(-8.552 \mathrm{kcal} / \mathrm{mol})$ formed the glide gscore energies (Table 1). Also, glide emodel scores were compatible with the glide gscore. The monophosphate and diphosphate interactions of favipiravir, remdesivir, ribavirin, and galidesivir were lower than triphosphate active forms, and the binding energy of triphosphate forms was lower than ATP. Based on these data, triphosphate forms of the prodrugs showed higher interactions. The interactions obtained as a result of molecular-docking studies of other intermediate metabolites are presented in Table 1. Interaction poses of GLT, RBT, FTP, RTP, and ATP at the NSP12-RNA strand active site are shown in Fig. 2.

\section{Molecular dynamics simulation}

Simulation of compound-structure complexes and apo-structure was carried out for $50 \mathrm{~ns}$. As the protein compound complexes' structural stability was examined, it was observed that the ATP-protein complex remained very stable under $0.1 \mathrm{~nm}$, and all the protein-ligand complexes were generally stable under $0.2 \mathrm{~nm}$. It was observed that the RMSD value of the ligand-free structure gradually increased and could not remain stable (Fig. 3). As the stability of protein-ligand complexes was examined based on residues, it was found that the fluctuation in the residues between 107 and 109 of the NSP12 chain in non-ligand apoprotein structure was higher than the protein-compound complexes. No significant change was observed in other chains and residues (Fig. 4). As the Radius of the gyration graph, which is the measure of protein compactness, was examined, it was seen that the ATP-bound structure was the most stable, followed by the FTP and RBT-bound structures (Fig. 5). As the protein compound interactions were examined at the end of $50 \mathrm{~ns}$, it was observed that ATP formed one hydrogen bond with Adenine and two hydrogen bonds with Uracil in RNA structure, and the compound also made one hydrogen bond with the Thr650. One of the $\mathrm{Mg}^{++}$atoms was observed to act as a metal acceptor, while the other $\mathrm{Mg}^{++}$atom was observed to participate in the ATP interaction with the attractive charge. In GLT residue interactions, it was observed that the adenine in the RNA structure formed a double hydrogen bond with GLT, and Arg555 formed a single hydrogen bond.

On the other hand, the compound created many negative interactions. As the RBT residue interaction was examined, it was observed that uracil in the RNA structure formed a hydrogen bond with the compound and formed a pi-pi-stacked interaction. Many hydrogens and salt bridge interactions were observed. In RTP residue interactions, it was observed that uracil in the RNA structure made double hydrogen bonding. The uracil in the structure of the other RNA chain made hydrogen bond. Many salt bridges with phosphate groups were observed (Figs. 6, 7). 
Table 1 RNA polymerase and RNA residues (PDB: 7BV2) interacting with galidesivir, ribavirin, remdesivir, favipiravir, and their metabolites through H-bonding, salt bridge, Pi-cation, and other interactions

\begin{tabular}{|c|c|c|c|c|}
\hline & Compound & Glide gscore & Glide emodel & Protein-RNA-ligand interactions \\
\hline GLS & & -6.187 & -58.201 & $\begin{array}{l}\text { ARG836, ARG858, PHE441, ASP845, LYS849, } \\
\text { LYS545, ALA547, ILE548, and P: A19, P: U18, } \\
\text { P: U17 }\end{array}$ \\
\hline GLM & $\mathrm{H}_{2} \mathrm{~N}$ & -7.855 & -87.796 & $\begin{array}{l}\text { Mg1004, ARG55, ARG555, VAL557, LYS545, } \\
\text { ASP618, ASP761, ASP760, SER682 and P: U20, } \\
\text { T: U10 }\end{array}$ \\
\hline GLD & H & -8.150 & -96.831 & $\begin{array}{l}\text { Mg1004, ILE548, ALA547, SER549, ASP618, } \\
\text { ASP760, ASP76, SER814, ARG55, ARG836 and } \\
\text { P: U20 }\end{array}$ \\
\hline GLT & & -8.994 & -107.152 & $\begin{array}{l}\text { Mg1004, LYS551, ILE548, ARG555, ALA547, } \\
\text { SER549, ARG836, SER814, ASP760, ASP761 } \\
\text { and P: U20 }\end{array}$ \\
\hline RBV & & -6.128 & -62.459 & $\begin{array}{l}\text { HIE439, SER814, LYS551, ALA550, ARG836, } \\
\text { GLU811, ASP760, ASP761 and P: U20 }\end{array}$ \\
\hline RBM & & -7.983 & -88.177 & $\begin{array}{l}\text { Mg1004, ARG555, VAL557, SER682, LYS545, } \\
\text { ASP760, ASP761 }\end{array}$ \\
\hline $\mathrm{RBD}$ & & -7.949 & -92.564 & $\begin{array}{l}\text { Mg1004, Mg1005, ARG555, ARG555, LYS545, } \\
\text { VAL557, SER682, ASP760, ASP761, GLU811, } \\
\text { SER814 and P: U20, T: U10 }\end{array}$ \\
\hline
\end{tabular}


Table 1 (continued)

\begin{tabular}{|c|c|c|c|c|}
\hline & Compound & Glide gscore & Glide emodel & Protein-RNA-ligand interactions \\
\hline RBT & & -9.820 & -101.863 & $\begin{array}{l}\text { Mg1004, SER553, LYS621, SER549, ILE548, } \\
\text { LYS551, HIE439, ALA550, ARG555, CYS622, } \\
\text { PRO620, TYR619, ASP760 and P: U20 }\end{array}$ \\
\hline RMD & & -7.755 & -109.151 & $\begin{array}{l}\text { ARG553, ARG555, ARG555, ALA554, VAL557, } \\
\text { CYS622, ASP623, ALA547, ILE548, SER549, } \\
\text { SER682, SER759, ASP760, ARG836, and P: } \\
\text { A19, P: A19 }\end{array}$ \\
\hline RMP & & -7.607 & -77.977 & $\begin{array}{l}\text { Mg1004, ARG555, VAL557, LYS45, SER682, } \\
\text { ASP760, ASP761 and P: U20, T: U10 }\end{array}$ \\
\hline RDP & & -7.312 & -90.081 & $\begin{array}{l}\text { Mg1004, ARG553, ARG555, ALA554, LYS551, } \\
\text { ALA550, SER549, ILE548, LYS545, ALA547, } \\
\text { SER814 and P: U20 }\end{array}$ \\
\hline RTP & & -9.278 & -108.979 & $\begin{array}{l}\text { Mg1004, LYS621, LYS551, ARG555, ARG553, } \\
\text { LYS545, VAL557, SER682, LYS545, ASP760, } \\
\text { CYS622, PRO620 and P: U20, T: U10 }\end{array}$ \\
\hline FPR & & -5.366 & -30.956 & $\begin{array}{l}\text { Mg1004, CYS622, ASP623, LYS621, TYR619, } \\
\text { ASP760 }\end{array}$ \\
\hline FRR & & -5.693 & -59.769 & $\begin{array}{l}\text { SER549, ILE548, ALA550, HIE439, ASP760, } \\
\text { ASP761, GLU811, SER814 and P: U20 }\end{array}$ \\
\hline
\end{tabular}


Table 1 (continued)

\begin{tabular}{|c|c|c|c|c|}
\hline & Compound & Glide gscore & Glide emodel & Protein-RNA-ligand interactions \\
\hline FMP & & -7.609 & -96.463 & $\begin{array}{l}\text { Mg1004, LYS545, ARG555, VAL557, SER682, } \\
\text { ASP760, ASP761 and P: U20, T: U10 }\end{array}$ \\
\hline FDP & & -8.223 & -97.563 & $\begin{array}{l}M g 1004, \text { SER682, ARG555, VAL557, LYS545, } \\
\text { ALA547, ASP760, ASP761, SER814 and P: U20 }\end{array}$ \\
\hline FTP & & -8.951 & -102.778 & $\begin{array}{l}\text { Mg1004, LYS551, LYS621, LYS551, LYS621, } \\
\text { ARG555, SER682, LYS545, ARG553, CYS622, } \\
\text { PRO620, TYR619 and P: U20 }\end{array}$ \\
\hline ATP & & -8.552 & -104.854 & $\begin{array}{l}\text { Mg1004, ARG55, ARG555, LYS545, VAL557, } \\
\text { SER682, THR680, THR687, ASN691, CYS622, } \\
\text { ASP623, ASP760, ASP761, SER759 and P: U20, } \\
\text { T: U10, T: A11 }\end{array}$ \\
\hline
\end{tabular}

Italic: salt bridge, bold: Hbond, red: Pi-cation İnteraction, normal: hydrophobic and other interactions

$G L S$ galidesivir, $G L M$ galidesivir monophosphate, $G L D$ galidesivir diphosphate, $G L T$ galidesivir triphosphate, $R B V$ ribavirin, $R B M$ ribavirin monophosphate, $R B D$ ribavirin diphosphate, $R B T$ ribavirin triphosphate, $R M D$ remdesivir, $R M P$ remdesivir monophosphate, $R D P$ remdesivir diphosphate, $R T P$ remdesivir triphosphate, $F P R$ favipiravir, $F R R$ favipiravir ribofuranose, $F M P$ favipiravir monophosphate, $F D P$ favipiravir diphosphate, FTP favipiravir triphosphate, $U$ uracil, $T$ thymine, $\mathrm{P}$ and T: chain

\section{Conclusion}

Since the RNA polymerase of SARS-CoV-2 is not present in humans and is important for the virus's survival, this enzyme's inhibition comes to the fore in drug research and development studies. Antiviral prodrugs such as favipiravir, remdesivir, galidesivir, and ribavirin inhibit the
RNA-dependent RNA polymerase of the virus, preventing the virus's replication. In this study, molecular docking studies of the prodrugs mentioned above and all their metabolites and molecular dynamics studies of active triphosphate metabolites were carried out. According to molecular docking results, triphosphate active metabolite 
Fig. 2 RBT (purple), GLT (yellow), RTP (green), FTP (blue), and ATP (gray) interactions in the active site of SARS-CoV-2 RNA polymerase

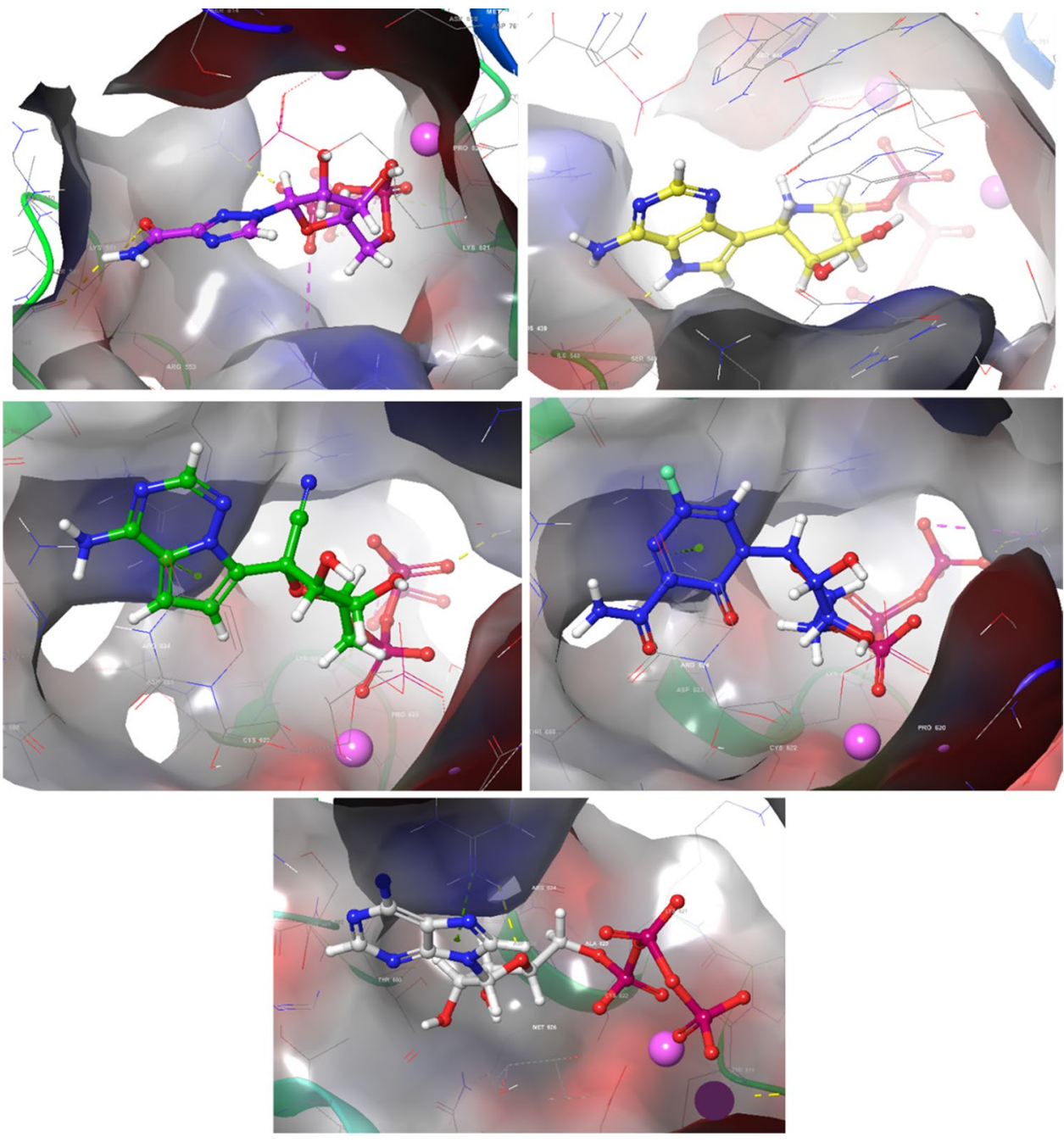

Backbone after Isq fit to Backbone

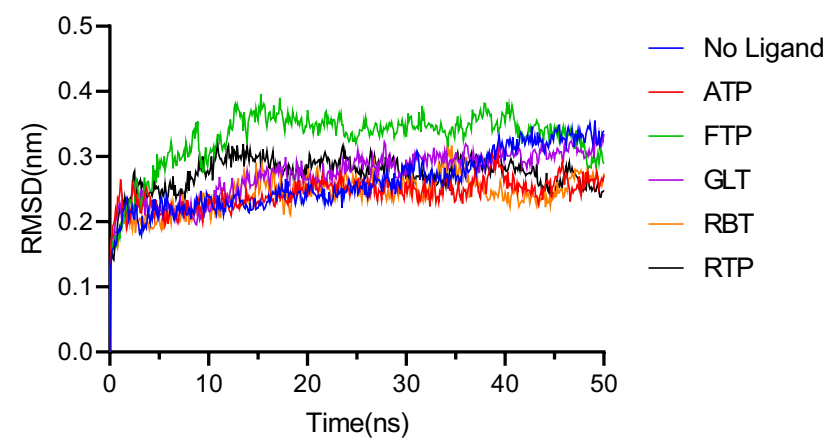

Fig. 3 Root-mean-square deviation (RMSD) analysis of the conformational stability of protein-ligand interactions throughout $50 \mathrm{~ns}$ compared to the non-liganded structure forms showed higher interaction than prodrug and other intermediate metabolites. We have also observed that these compounds increase the complex's stability and validate the molecular docking results with the RMSD, RMSF, and radius of gyration analyses we obtained with MD simulation. For RNA polymerase inhibition to occur, they must form a salt bridge of $\mathrm{Mg}^{++}$with the first ribofuranose linked phosphate group and interact with Hie439, Lys545, Ile 548, Ser549, Arg553, Arg555, Val557, Lys621, Cys622, Ser682, Asn691, and Asp760 required. It must also form a hydrogen bond with U20 in the RNA helix. In computer-aided drug design studies, it should not be overlooked that it is indispensable for both the inhibitors' active metabolites and the inhibition of interacting with the RNA strand. 


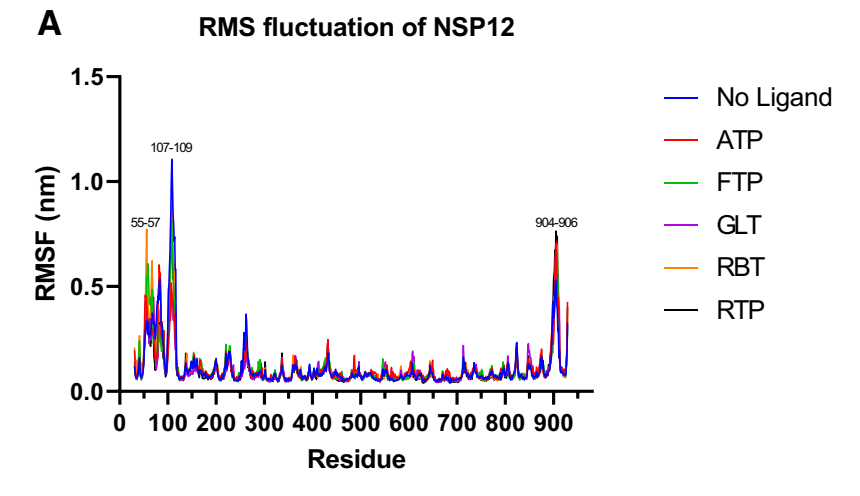

B RMS fluctuation of NSP8
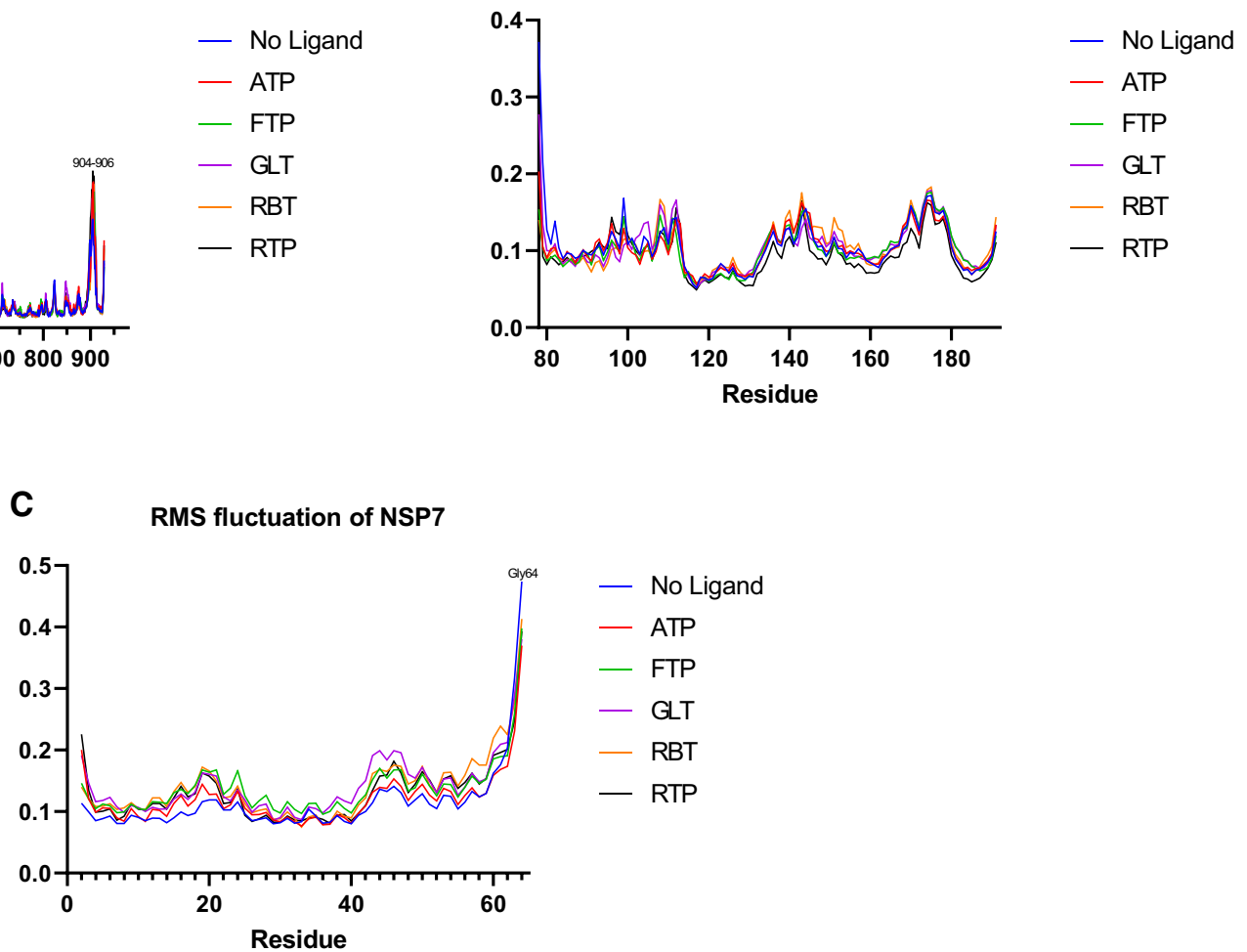

Fig. 4 Root-mean-square fluctuation (RMSF) analysis of structures with and without ligand throughout $50 \mathrm{~ns}$

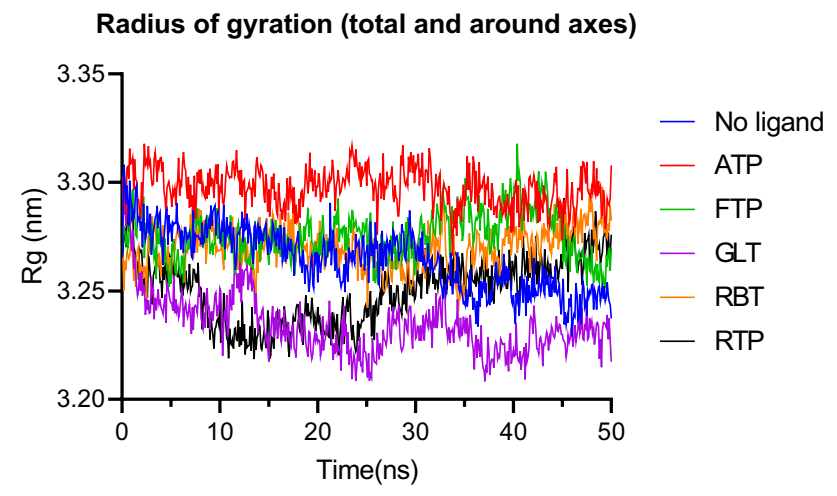

Fig. 5 The radius of gyration graph of structures with and without ligands over $50 \mathrm{~ns}$ 
Fig. 6 Protein-compound interactions after $50 \mathrm{~ns}$ MD Simulations

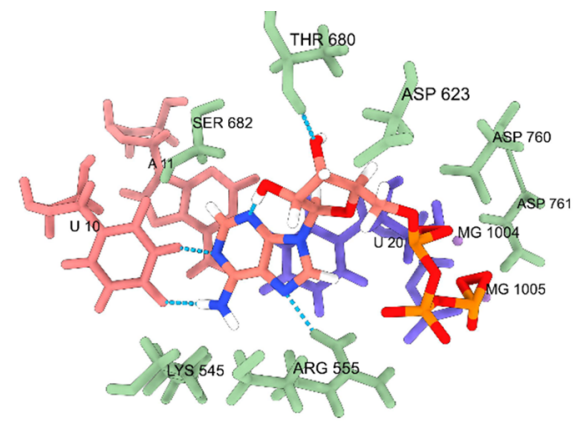

ATP

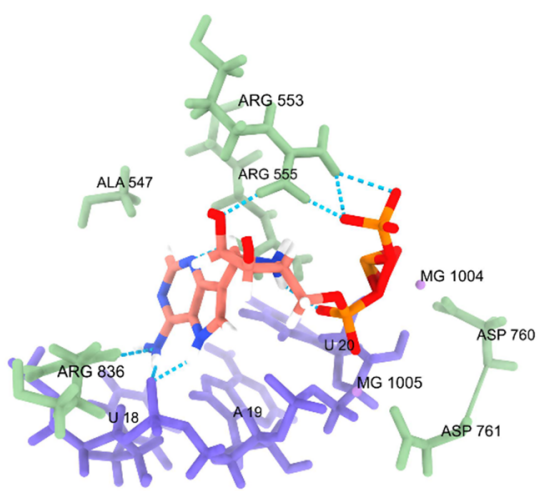

GLT
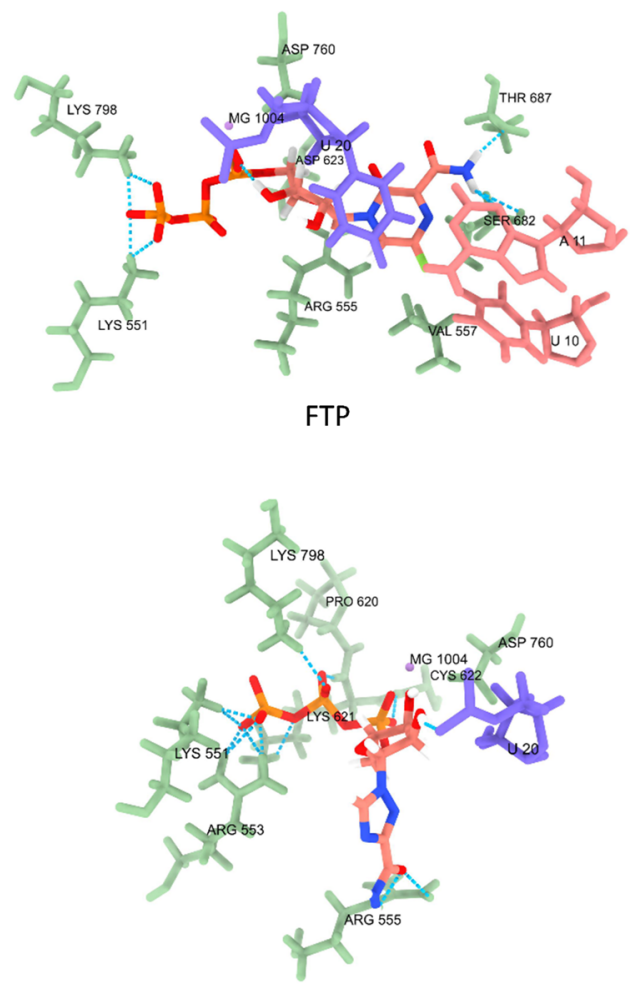

RBT

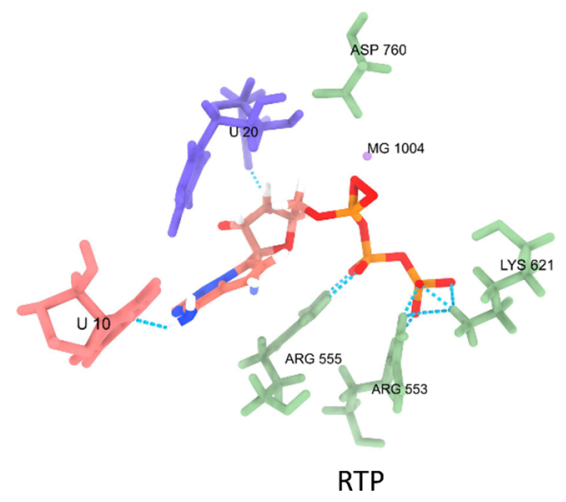



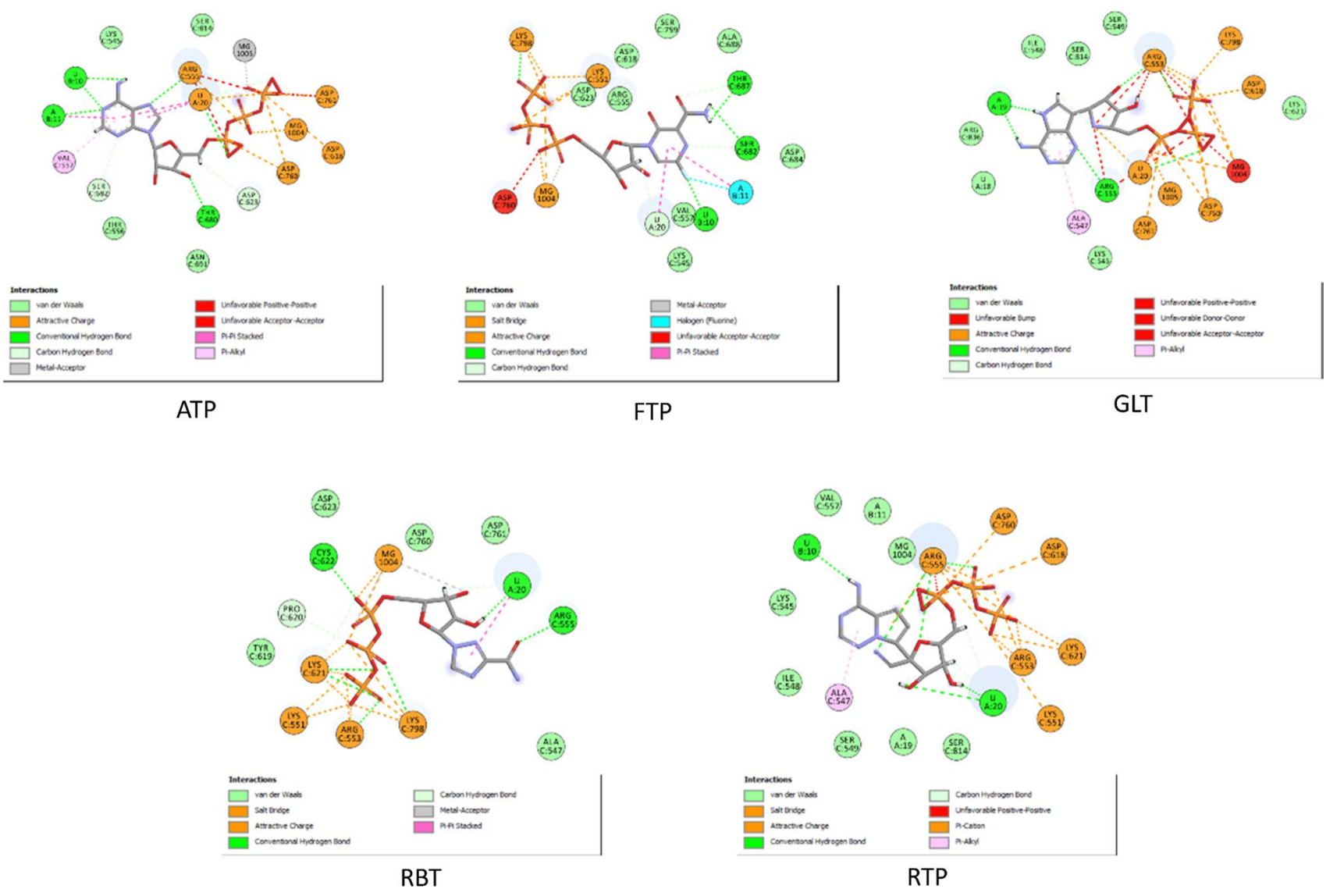

Fig. 7. 2D Diagram of Protein-compound interactions after $50 \mathrm{~ns}$ MD Simulations

Acknowledgements All molecular dynamics simulations reported were performed utilizing TÜBITTAK (The Scientific and Technological Research Council of Turkey), ULAKBİM (The Turkish Academic Network and Information Center), and the High Performance and Grid Computing Center (TRUBA resources).

\section{References}

1. Romano M, Ruggiero A, Squeglia F, Maga G, Berisio R (2020) A Structural view of SARS-CoV-2 RNA replication machinery: RNA synthesis. Proofreading Final Cap Cells 9(5):1267. https:// doi.org/10.3390/cells9051267

2. Chan JF-W, Kok K-H, Zhu Z, Chu H, ToYuan KK-WS, Yuen K-Y (2020) Genomic characterization of the 2019 novel humanpathogenic coronavirus isolated from a patient with atypical pneumonia after visiting Wuhan. Emerg Microbes Infect 9(1):221-236. https://doi.org/10.1080/22221751.2020.1719902

3. Naik VR, Munikumar M, Ramakrishna U, Srujana M, Goudar G, Naresh P, KumarHemalatha BNR (2020) Remdesivir (GS5734 ) as a therapeutic option of 2019-nCOV main protease-in silico approach. J Biomol Struct Dyn. https://doi.org/10.1080/ 07391102.2020.1781694

4. Cucinotta D, Vanelli M (2020) WHO declares COVID-19 a pandemic. Acta Biomed Ateneo Parmense 91(1):157-160. https:// doi.org/10.23750/abm.v91i1.9397
5. Organization WHO (2020) Coronavirus disease 2019 (COVID19): situation report, 82

6. Guan W-j, Ni Z-y, Hu Y, Liang W-h, Ou C-q, He J-x, Liu L, ShanLei HC-1, Hui DS (2020) Clinical characteristics of coronavirus disease 2019 in China. N Engl J Med 82(18):1708-1720. https://doi.org/10.1056/NEJMoa2002032

7. Wang Z, Yang B, Li Q, WenZhang LR (2020) Clinical features of 69 cases with coronavirus disease 2019 in Wuhan. China Clin Infect Dis 71(15):769-777. https://doi.org/10.1093/cid/ciaa272

8. Wang C, Horby PW, HaydenGao FGGF (2020) A novel coronavirus outbreak of global health concern. Lancet 395(10223):470 473. https://doi.org/10.1016/S0140-6736(20)30185-9

9. Guan W, Ni Z, Hu Y, Liang W, Ou C, He J, Liu L, Shan H, LeiHui CD (2019) Clinical characteristics of coronavirus disease. N Engl J Med 382:1708-1720. https://doi.org/10.1056/NEJMoa2002032

10. Burrell CJ, HowardMurphy CRFA (2016) Fenner and white's medical virology. Academic Press, London

11. Zhou P, Yang X-L, Wang X-G, Hu B, Zhang L, Zhang W, Si H-R, Zhu Y, LiHuang BC-L (2020) A pneumonia outbreak associated with a new coronavirus of probable bat origin. Nature 579(7798):270-273. https://doi.org/10.1038/s41586-020-2012-7

12. Xu X, Chen P, Wang J, Feng J, Zhou H, Li X, ZhongHao WP (2020) Evolution of the novel coronavirus from the ongoing Wuhan outbreak and modeling of its spike protein for risk of human transmission. Sci China Life Sci 63(3):457-460. https:// doi.org/10.1007/s11427-020-1637-5

13. Li B, Si H-R, Zhu Y, Yang X-L, Anderson DE, Shi Z-L, WangZhou L-FP (2020) Discovery of bat coronaviruses through 
surveillance and probe capture-based next-generation sequencing. Msphere 5(1):1-11. https://doi.org/10.1128/mSphere.00807-19

14. Lan J, Ge J, Yu J, Shan S, Zhou H, Fan S, Zhang Q, Shi X, Wang Q, Zhang L (2020) Structure of the SARS-CoV-2 spike receptor-binding domain bound to the ACE2 receptor. Nature 581(7807):215-220. https://doi.org/10.1038/s41586-020-2180-5

15. Wang Q, Zhang Y, Wu L, Niu S, Song C, Zhang Z, Lu G, Qiao C, $\mathrm{Hu}$ Y, Yuen K-Y (2020) Structural and functional basis of SARSCoV-2 entry by using human ACE2. Cell 181(4):894-904. https:// doi.org/10.1016/j.cell.2020.03.045

16. Hoffmann M, Kleine-Weber H, Krüger N, Mueller MA, DrostenPöhlmann CS (2020) The novel coronavirus 2019 (2019-nCoV) uses the SARS-coronavirus receptor ACE2 and the cellular protease TMPRSS2 for entry into target cells. BioRxiv. https://doi. org/10.1101/2020.01.31.929042

17. Ayten O, Özdemir C, Aktürk ÜA, Şen N (2020) Potential treatment of COVID-19. Eurasian J Pulmonol 22(4):35. https://doi. org/10.4103/ejop.ejop_61_20

18. Casadevall A, Pirofski L-A (2020) The convalescent sera option for containing COVID-19. J Clin Invest 130(4):1545-1548. https://doi.org/10.1172/JCI138003

19. Jorgensen SC, Kebriaei R, Dresser LD (2020) Remdesivir: review of pharmacology, pre-clinical data and emerging clinical experience for COVID-19. Pharmacotherapy 40(7):659-671. https://doi.org/10.1002/phar.2429

20. Wiemer AJ (2020) Metabolic efficacy of phosphate prodrugs and the remdesivir paradigm. ACS Pharmacol Transl Sci 3(4):613-626. https://doi.org/10.1021/acsptsci.0c00076

21. Sangawa H, Komeno T, Nishikawa H, Yoshida A, Takahashi K, Nomura N, Furuta Y (2013) Mechanism of action of T-705 ribosyl triphosphate against influenza virus RNA polymerase. Antimicrob Agents Chemother 57(11):5202-5208. https://doi. org/10.1128/AAC.00649-13

22. Santos J, Brierley S, Gandhi MJ, Cohen MA, Moschella PC, Declan AB (2020) Repurposing therapeutics for potential treatment of SARS-CoV-2: a review. Viruses 12(7):1-19. https://doi. org/10.3390/v12070705

23. Kandimalla R, John A, Abburi C, Vallamkondu J, Reddy PH (2020) Current status of multiple drug molecules, and vaccines: an update in SARS-CoV-2 therapeutics. Mol Neurobiol 57:41064116. https://doi.org/10.1007/s12035-020-02022-0

24. Hashemian SM, Farhadi T, Velayati AA (2020) A review on remdesivir: a possible promising agent for the treatment of COVID19. Drug Des Devel Ther 14:3215-3222. https://doi.org/10.2147/ DDDT.S261154

25. Wang Y, Anirudhan V, Du R, Cui Q, Rong L (2021) RNA-dependent RNA polymerase of SARS-CoV-2 as a therapeutic target. J Med Virol 93(1):300-310. https://doi.org/10.1002/jmv.26264

26. Abet V, Filace F, Recio J, Alvarez-Builla J, Burgos C (2017) Prodrug approach: an overview of recent cases. Eur J Med Chem 127:810-827. https://doi.org/10.1016/j.ejmech.2016.10.061

27. Ghosh GC, Nakada N, Yamashita N, Tanaka H (2010) Oseltamivir carboxylate, the active metabolite of oseltamivir phosphate (Tamiflu), detected in sewage discharge and river water in Japan. Environ Health Perspect 118(1):103-107. https://doi.org/10.1289/ ehp.0900930

28. Chauhan DS, Prasad R, Srivastava R, Jaggi M, Chauhan SC, Yallapu MM (2020) Comprehensive review on current interventions, diagnostics, and nanotechnology perspectives against SARSCoV-2. Bioconjug Chem 31(9):2021-2045. https://doi.org/10. 1021/acs.bioconjchem.0c00323

29. Artese A, Svicher V, Costa G, Salpini R, Di Maio VC, Alkhatib M, Ambrosio FA, Santoro MM, Assaraf YG, Alcaro S (2020) Current status of antivirals and druggable targets of SARS CoV-2 and other human pathogenic coronaviruses. Drug Resist Updat 53:100721. https://doi.org/10.1016/j.drup.2020.100721
30. Sareen K, Bose R, Singh R, Boddu L (2020) Treatment of COVID-19. Praxis Undergraduate Med Res J 3:56-64

31. Ju J, Kumar S, Li X, Jockusch S, Russo JJ (2020) Nucleotide analogues as inhibitors of viral polymerases. BioRxiv. https://doi. org/10.1101/2020.01.30.927574

32. Dömling A, Gao L (2020) Chemistry and biology of SARSCoV-2. Chem 6(6):1283-1295. https://doi.org/10.1016/j.chempr. 2020.04.023

33. De LG, Clercq E (2020) Therapeutic options for the 2019 novel coronavirus (2019-nCoV). Nat Rev Drug Discov 19:149-150. https://doi.org/10.1038/d41573-020-00016-0

34. Abuo-Rahma GE-DA, Mohamed MF, Ibrahim TS, Shoman ME, Abd SE, El-Baky RM (2020) Potential repurposed SARS-CoV-2 (COVID-19) infection drugs. RSC Adv 10(45):26895-26916. https://doi.org/10.1039/D0RA05821A

35. Akhtar MJ (2020) COVID19 inhibitors: a prospective therapeutics. Bioorg Chem 101:104027. https://doi.org/10.1016/j.bioorg. 2020.104027

36. Wang Y, Anirudhan V, Du R, Cui Q, Rong L (2020) RNA-dependent RNA polymerase of SARS-CoV-2 as a therapeutic target. J Med Virol 93(1):300-310. https://doi.org/10.1002/jmv.26264

37. Du YX, Chen XP (2020) Favipiravir: pharmacokinetics and concerns about clinical trials for 2019-nCoV infection. Clin Pharm 108(2):242-247. https://doi.org/10.1002/cpt.1844

38. Giovane RA, Rezai S, Cleland E, Henderson CE (2020) Current pharmacological modalities for management of novel coronavirus disease 2019 (COVID-19) and the rationale for their utilization: a review. Rev Med Virol 30(5):1-4. https://doi.org/10.1002/rmv. 2136

39. Chien M, Anderson TK, Jockusch S, Tao C, Li X, Kumar S, Russo JJ, Kirchdoerfer RN, Ju J (2020) Nucleotide analogues as inhibitors of SARS-CoV-2 polymerase, a key drug target for COVID-19. J Proteome Res 19(11):4690-4697. https://doi.org/10.1021/acs. jproteome.0c00392

40. Eastman RT, Roth JS, Brimacombe KR, Simeonov A, Shen M, Patnaik S, Hall MD (2020) Remdesivir: a review of its discovery and development leading to emergency use authorization for treatment of COVID-19. ACS Cent Sci 6(5):672-683. https://doi.org/ 10.1021/acscentsci.0c00489

41. Neerukonda SN, Katneni U (2020) A review on SARS-CoV-2 virology, pathophysiology, animal models, and anti-viral interventions. Pathogens 9(6):1-22. https://doi.org/10.3390/pathogens9 060426

42. Grein J, Ohmagari N, Shin D, Diaz G, Asperges E, Castagna A, Feldt T, Green G, GreenLescure MLF-X (2020) Compassionate use of remdesivir for patients with severe Covid-19. N Engl J Med 382(24):2327-2336. https://doi.org/10.1056/NEJMoa2007016

43. Elfiky AA (2020) Ribavirin, remdesivir, sofosbuvir, galidesivir, and tenofovir against SARS-CoV-2 RNA dependent RNA polymerase (RdRp): a molecular docking study. Life Sci 253:117592. https://doi.org/10.1016/j.lfs.2020.117592

44. Naik B, Gupta N, Ojha R, Singh S, PrajapatiPrusty VKD (2020) High throughput virtual screening reveals SARS-CoV-2 multitarget binding natural compounds to lead instant therapy for COVID-19 treatment. Int J Biol Macromol 160:1-17. https://doi. org/10.1016/j.ijbiomac.2020.05.184

45. Elfiky AA (2020) SARS-CoV-2 RNA dependent RNA polymerase (RdRp) targeting: an in silico perspective. J Biomol Struct Dyn. https://doi.org/10.1080/07391102.2020.1761882

46. Koulgi S, Jani V, Uppuladinne MV, SonavaneJoshi UR (2020) Remdesivir-bound and ligand-free simulations reveal the probable mechanism of inhibiting the RNA dependent RNA polymerase of severe acute respiratory syndrome coronavirus 2. RSC Adv 10(45):26792-26803. https://doi.org/10.1039/D0RA04743K

47. Kar P, Sharma NR, Singh B, Sen A, Roy A (2020) Natural compounds from Clerodendrum spp. as possible therapeutic 
candidates against SARS-CoV-2: An in silico investigation. J Biomol Struct Dyn. https://doi.org/10.1080/07391102.2020.1780947

48. Alamri MA, Altharawi A, Alabbas AB, AlossaimiAlqahtani MASM (2020) Structure-based virtual screening and molecular dynamics of phytochemicals derived from Saudi medicinal plants to identify potential COVID-19 therapeutics. Arab J Chem 13(9):7224-7234. https://doi.org/10.1016/j.arabjc.2020.08.004

49. Yin W, Mao C, Luan X, Shen D-D, Shen Q, Su H, Wang X, Zhou F, ZhaoGao WM (2020) Structural basis for inhibition of the RNA-dependent RNA polymerase from SARS-CoV-2 by remdesivir. Science 368(6498):1499-1504. https://doi.org/10.1126/ science.abc 1560

50. Pettersen EF, Goddard TD, Huang CC, Couch GS, Greenblatt DM, MengFerrin ECTE (2004) UCSF Chimera-a visualization system for exploratory research and analysis. J Comput Chem 25(13):1605-1612. https://doi.org/10.1002/jcc.20084

51. Pradhan D, Priyadarshini V, Munikumar M, Swargam S, Umamaheswari A, Bitla A (2014) Para-(benzoyl)-phenylalanine as a potential inhibitor against LpxC of Leptospira spp.: homology modeling, docking, and molecular dynamics study. J Biomol Struct Dyn 32(2):171-185. https://doi.org/10.1080/07391102. 2012.758056

52. Priyadarshini V, Pradhan D, Munikumar M, Swargam S, UmamaheswariRajasekhar AD (2014) Genome-based approaches to develop epitope-driven subunit vaccines against pathogens of infective endocarditis. J Biomol Struct Dyn 32(6):876-889. https://doi.org/10.1080/07391102.2013.795871
53. Munikumar M, Krishna VS, Reddy VS, Rajeswari B, SriramRao DMV (2018) In silico design of small peptides antagonist against leptin receptor for the treatment of obesity and its associated immune-mediated diseases. J Mol Graph 82:20-36. https://doi. org/10.1016/j.jmgm.2018.04.002

54. Salomon-Ferrer R, CaseWalker DARC (2013) An overview of the Amber biomolecular simulation package. Wiley Interdiscip Rev Comput Mol Sci 3(2):198-210. https://doi.org/10.1002/wcms. 1121

55. Da Silva AWS, Vranken WF (2012) ACPYPE-Antechamber python parser interface. BMC Res Notes 5(1):367. https://doi. org/10.1186/1756-0500-5-367

56. Abraham MJ, Murtola T, Schulz R, Páll S, Smith JC, HessLindahl BE (2015) GROMACS: High performance molecular simulations through multi-level parallelism from laptops to supercomputers. SoftwareX 1-2:19-25. https://doi.org/10.1016/j.softx.2015.06. 001

57. Lindorff-Larsen K, Piana S, Palmo K, Maragakis P, Klepeis JL, DrorShaw RODE (2010) Improved side-chain torsion potentials for the Amber ff99SB protein force field. Proteins 78(8):19501958. https://doi.org/10.1002/prot.22711

58. Zhang L, Zhou R (2020) Binding mechanism of remdesivir to SARS-CoV-2 RNA dependent RNA polymerase. Preprints 1-18. https://doi.org/10.20944/preprints202003.0267.v1

Publisher's note Springer Nature remains neutral with regard to jurisdictional claims in published maps and institutional affiliations. 